\title{
Development of Cortical Integration of Visual Stimuli in Very Preterm Infants
}

\author{
Zores Ca,b, Marchal Aa, Davy Ma, Pebayle T', Astruc Da, Dufour A ${ }^{b}$, Kuhn Pa,c \\ a Médecine et Réanimation du nouveau-né, Service de Pédiatrie 2, Pôle Médico-Chirurgical Pédiatrique, Hôpital de Hautepierre, \\ Centre Hospitalier Universitaire de Strasbourg, Strasbourg, France. \\ ${ }^{\text {b}}$ Laboratoire de Neurosciences Cognitives et Adaptatives, UMR 7364 CNRS/Université de Strasbourg, Strasbourg, France \\ c Institut des neurosciences cellulaires et intégratives, Déterminants moléculaires de la douleur Strasbourg, France
}

\section{Introduction}

Very preterm infants (VPI) are exposed to atypical visual stimuli in the hospital. They are able to exhibit physiological and behavioural responses to ecological visual stimuli in the NICU from 28 weeks post-menstrual age (PMA). ${ }^{1,2}$ However, little is known about their cortical responses to light level changes and the development of this cortical integration until term post-menstrual age (PMA).

\section{Aims}

We aimed to evaluate the cortical responses of VPIs to light level changes of different intensities. Our goal was also to follow longitudinally the development of the cortical integration of these stimuli in VPIs until term corrected age and to compare them to the responses of full-term newborn infants.

\section{Methods}

We included 25 term newborn infants and 26 VPI at Strasbourg University Hospital (France). The VPIs were studied at three distinct ages: $32(n=21), 36(n=23)$ and $40(n=21)$ weeks PMA. Two different light stimulations (100 and 300 lux above basal light level) were presented in a random order for duration of
5 seconds. Oxyhemoglobin and deoxyhemoglobin changes were recorded by multi-channel near infrared spectroscopy $\left(\mathrm{NIRx}^{\circ}\right)$. Optodes were positioned using the EEG 10-20 classification to explore the areas of interest. ${ }^{3}$ The first regions of interest were occipital (O) visual areas: OI (middle and superior occipital gyri), OII (inferior occipital gyrus and calcarine sulcus), pariétooccipital (PO: middle occipital gyrus and angular gyrus). We also recorded hemodynamic changes in frontal areas (F: middle frontal gyrus) and prefrontal areas ( $\mathrm{pF}$ : middle frontal gyrus and orbitary frontal gyri). After a specific pretreatment of the data we carefully rejected artifacts. Oxyhemoglobin variations were analyzed from baseline (10 s) to $25 \mathrm{~s}$ post-stimulation by ANOVA for repeated measure.

\section{Results/Findings}

A 100 lux stimulus triggered a significant increase in oxyhemoglobin $(0.6$ to $1.4 \mu \mathrm{mol} / \mathrm{L})$ in visual areas as early as 36 weeks PMA ( $\mathrm{p}<0.01$ ). This response was also fully present in full-term infants but less present at 32 weeks PMA. Increases in oxyhemoglobin were also noted in frontal areas, but only in VPIs at 40 weeks PMA. All these results are shown in Table 1. At term corrected age, the profile of responses of VPIs and full-term new-

TABLE 1. Cerebral activation in response to a 100 lux light stimulation in different groups

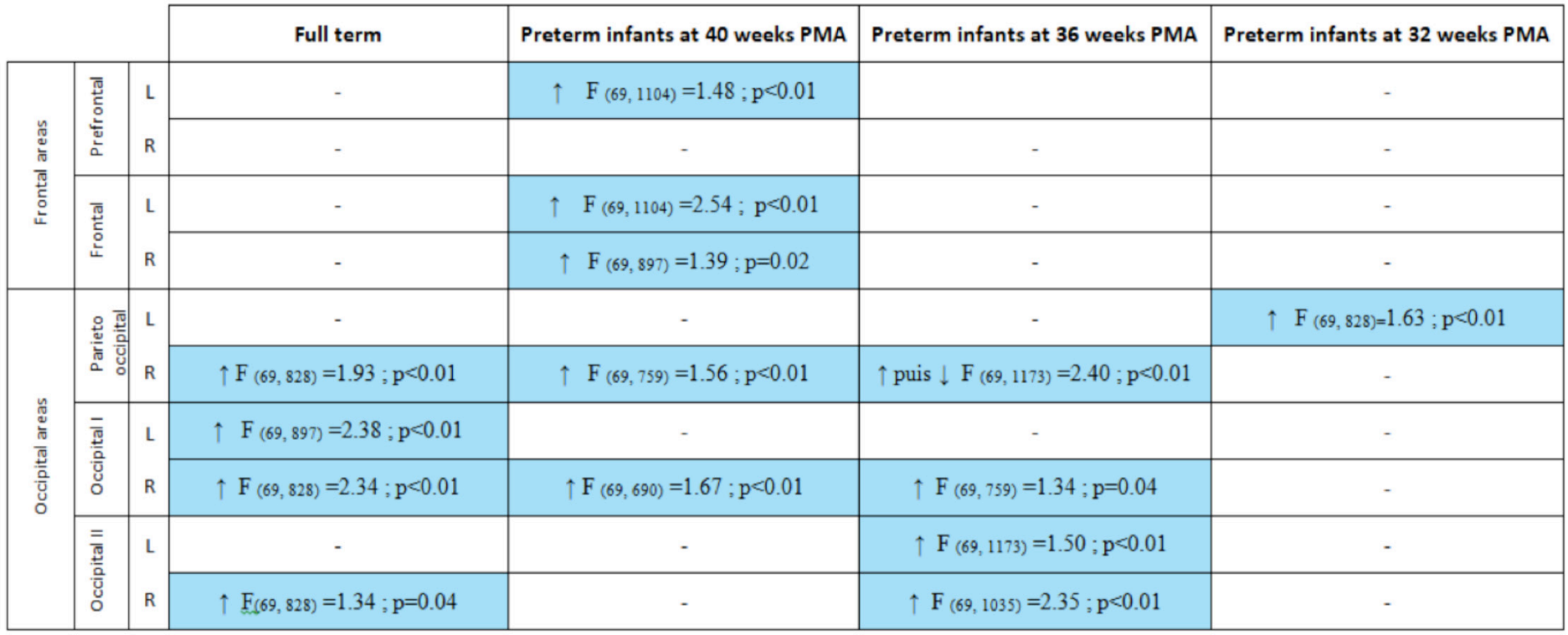

The arrow shows the sense of variation of oxyhemoglobin; L: left; $R$ : right

16 • 2019 • Developmental Observer 
FIGURE 1. Cerebral responses measured in one occipital area (left OI) at 40 weeks PMA

after a 300 lux light stimulation

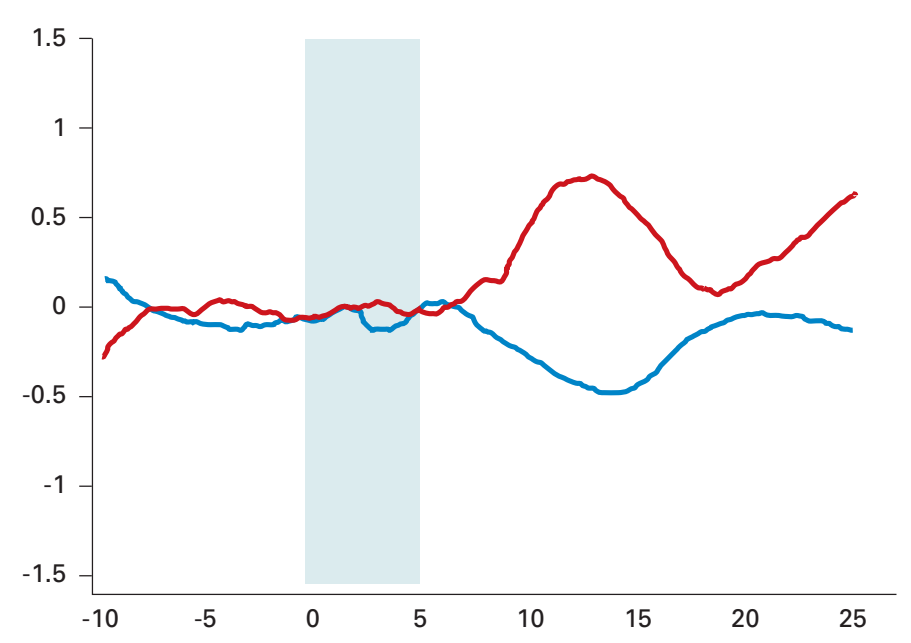

Variation of oxyhemoglobin (red line) and deoxyhemoglobin (blue line)

borns were different in both occipital and frontal areas $(\mathrm{p}<0.05)$.

A 300 lux stimulus triggered cerebral activation mainly in the frontal and prefrontal areas whatever the age of the infants. An increase in oxyhemoglobin was present at 32 weeks of PMA in the frontal and prefrontal areas and in the occipital areas from 36 weeks of PMA. An oxyhemoglobin decrease was also seen in some regions of interest. We present in Figure 1 the responses measured in one occipital area at 40 weeks PMA.

\section{Conclusion}

Cerebral hemodynamic responses to 100 and 300 lux stimuli were inconsistant but present in at least one visual area in full-term infants and as early as 36 weeks PMA in VPIs. At 32 weeks PMA, variations of oxyhemoglobin were more inconsistant, more moderate and less diffuse. They were mainly found for higher intensities. The pattern of cortical activation for one stimulation was variable in the different age groups. We observed different profiles of oxyhemoglobin responses in term and VPIs at 40 weeks PMA. These results suggest a possible impact of premature birth and of the early visual environment on the developmental trajectory of the visual system with potential alteration of the neuronal network. These results support the need for better attention to light level changes during hospitalization. ${ }^{4}$ The long term effects of the early visual environment should be evaluated further.

References

1. Zores C, Dufour A, Pebayle T, Langlet C, Astruc D, Kuhn P. Very preterm infants can detect small variations in light levels in incubators. Acta Paediatrics 2015;104:1005-11.

2. Zores C, Dufour A, Pebayle T, Dahan I, Astruc D, Kuhn P. Observational study found that even small variations in light can wake up very preterm infants in a neonatal intensive care unit. Acta Paediatrics 2018;107:1191-97.

3. Kabdebon C, Leroy F, Simmonet H, Perrot M, Dubois J, Dehaene-Lambertz G. Anatomical correlations of the international 10-20 sensor placement system in infants. Neuroimage 2014;99:342-56.

4. White RE. Defining the optimal sensory environment in the NICU: an elusive task. Acta Paediatrics 2018;107:1112-1112. 